\title{
Refinery-wide control loop performance assessment
}

\author{
N.F. Thornhill ${ }^{\mathrm{a}, *}$, M. Oettinger ${ }^{\mathrm{b}, 1}$, P. Fedenczuk ${ }^{\mathrm{c}}$ \\ ${ }^{a}$ Department of Electronic and Electrical Engineering, University College London, Torrington Place, London WC1E 7JE, UK \\ ${ }^{\mathrm{b}}$ BP Refinery (Kwinana) Proprietary Ltd., Mason Road, Kwinana, WA 6167, Australia \\ ${ }^{\mathrm{c}}$ BP Oil, Grangemouth Refinery Ltd., PO Box 30, Bo'ness Road, Grangemouth, FK3 9XQ, UK
}

Received 1 October 1997; received in revised form 15 June 1998; accepted 1 July 1998

\begin{abstract}
This paper discusses the application of control loop performance assessment (CLPA) in a refinery setting. The CLPA algorithm has several parameters that have to be adjusted correctly to give the best results. Procedures are described for selecting these parameters which make it feasible to implement the algorithm on a refinery-wide scale. We report practical experiences with the use of the techniques. (C) 1999 Elsevier Science Ltd. All rights reserved.
\end{abstract}

Keywords: Control loop performance assessment; Refinery-wide; Implementation; Guidelines; Practical experience

\section{Introduction}

Previous studies of the performance of single-inputsingle-output (SISO) control loops have shown that reasons for poor performance of basic SISO loops include both poor tuning and equipment problems such as sticking valves [1-3]. Traditional methods used for performance assessment include inspections of control loop hardware, logging of the percentage of time control loops are in AUTO mode and calculation of the mean and standard deviation of the controlled process variables. Improved control yields commercial benefits [4] while other benefits of a systematic assessment include the ease of maintenance of basic control functions and the reduction in man-hours in the troubleshooting of control problems.

A vision for controller performance monitoring has been set out by D. Kozub of the Shell Development Company [5]. He has set out challenges for the research community to develop tools and methodologies for automated technology. Not only is performance information sought; ideally the monitoring system should aid the prioritisation of control problems. His paper also sets out goals for the diagnosis of the reasons for loss of performance once a poorly performing loop has been

\footnotetext{
* Corresponding author. Tel.: +44-171-387-7050; fax:+44-1713874350; e-mail: n.thornhill@ee.ucl.ac.uk

${ }^{1}$ Present affiliation: Senior Area Process Engineer, AMPOL Refineries (NSW) Pty Ltd, Taren Point, NSW 2229, Australia.
}

detected, for example by determination of dynamic response characteristics such as the extent of underdamped or oscillatory behaviour.

Some quantitative measures of performance such as settling time and time integral measures need a special test of the loop, for example an examination of its step response. A new approach to performance assessment has been developed by Harris [6], Desborough and Harris [7] and Stanfelj et al. [8]. An advantage of their methods is that they provide figures of merit for the performance which can be derived during periods of normal running without taking loops off-line for special tests. These methods are becoming widely implemented in the petrochemical and chemical sectors [5,8-10] and also in the pulp and paper industry [11-14]. Recent work $[15,16]$ has been addressing some of the challenges laid down by Kozub and Garcia [9] and Kozub [5]. These authors described large scale implementation of SISO performance assessment and diagnosis in an expert system setting. They made use of an expert system to achieve on-line monitoring and a user interface that allowed a user to view detailed performance information for an individual loop. Control loop diagnosis has also been examined by [17]; flow charts based upon certain signatures in the controller data guide decisions about a diagnosis of a tuning or equipment fault.

The key variable for CLPA when applied to feedback control is the controller error, $y$, given by the difference between the set point $(s p)$ and the process variable $(p v)$. If the loop is performing well it should reject disturbances 
and the process variable should track the set point. These requirements imply that the controller error should have no predictable component. There should not, for example, be a steady state offset or any predictable oscillation. Harris [6] devised an index based upon the residuals between the measured controller error denoted by $y$ and a forward prediction, $\hat{y}$ :

$r(i)=y(i)-\hat{y}(i)$

Because of the dynamic nature of the process and of the controller itself it takes a little time for the controller to achieve rejection of a disturbance or to bring the process to its set point. Thus it is necessary to determine how predictable the controller error is beyond some suitable time horizon. In a loop that is performing well the controller error has little predictability beyond that time horizon and the controller error contains only the random noise represented by the residuals. In a poorly performing loop, however, the ratio between the random residuals and the controller error is less than unity because a predictable component is present. That is, a loop which is performing well has a low signal to noise ratio while a poorly performing loop has a high one. Desborough and Harris [7] defined an index for SISO control loop performance, given by the expression below in which $\sigma_{r}^{2}$ is the variance of the (zero mean) residuals and $m s e\left(y_{i}\right)$ is the mean square value of the controller error. A poorly performing loop has a value of the index close to 1 while the index for a good loop is close to 0 :

$1-\frac{\sigma_{r}^{2}}{m s e\left(y_{i}\right)}$

The requirement for the model for $\hat{y}$ is that it is capable of capturing predictable features in the controller error sequence. Desborough and Harris [7] discuss suitable models and show that an autoregressive time series model that makes predictions $b$ steps ahead is suitable. Other papers $[6,8]$ discuss various alternative model structures. The model used in this work has $m$ autoregressive terms and a constant term $a_{o}$.

$$
\begin{aligned}
\hat{y}(i+b)=a_{0} & +a_{1} y(i)+a_{2} y(i-1) \\
& +a_{3} y(i-2)+\ldots . .+a_{m} y(i-m+1)
\end{aligned}
$$

The above model is fitted to an ensemble of $n$ samples of the controller error using a least squares fit procedure. The matrix with the following structure is denoted by $\mathbf{X}$ :

$$
\left(\begin{array}{cccc}
y(m) & . . & y(1) & 1 \\
y(m+1) & . . & y(2) & 1 \\
. . & . . & . . & . \\
y(n-b) & . . & y(n-b-m+1) & 1
\end{array}\right)
$$

and the best fit coefficients for the vector $\left(a_{m} a_{m-1} \ldots . . a_{0}\right)^{T}$ are given by the expression:

$\mathbf{a}=\left(\mathbf{X}^{T} \mathbf{X}\right)^{-1} \mathbf{X}^{T} \underline{\mathbf{Y}}$

where $\underline{\mathbf{Y}}$ is $(y(m+b) . . y(n))^{T}$. The work presented here used an implementation of the algorithm in MATLAB $^{\text {TM }}$ (The MathWorks, Natick, MA.) provided by Sadowski and Associates (Richfield, OH, USA).

The contribution of this paper is to provide guidelines for large scale implementation of CLPA technology. It provides recommendations for the sampling interval and the parameters $n, m$ and $b$ that may be used as defaults in all refineries so that the algorithm of Desborough and Harris [7] can be automated to give routine judgements on the performance of most refinery loops. The use of default settings for the parameters makes a refinery-wide implementation feasible and therefore addresses the goal of Kozub [5] for the development of automated on-line CLPA technology. The paper also presents methods that will help a control engineer to select values of the parameters for optimisation of the algorithm in special cases where the defaults are not suitable. It reports practical experiences gained with SISO refinery control loops and is illustrated with 12 of them.

The next section of the paper explains how the default values were selected for the sampling interval and the parameters $n, m$ and $b$. Numerical implementation is also discussed, and in particular the paper investigates the performance of the CLPA method with archived data that have been subject to smoothing and compression. The performance of one loop is repeatedly assessed at different controller tuning settings in the section 'Tuning trials'. The aim of the 'Discussion' section is to give some insights into how to interpret the results. Finally, the 'Conclusions' section summarises key features of the work and focuses on the benefits it offers to refinery control engineers.

\section{Application on a refinery-wide scale}

\subsection{A strategy for application to a large plant}

In a refinery there are typically more than 1000 basic SISO feedback loops. An automated CLPA technique needs a means of providing an autoregressive model [Eq. (2)] for every loop. Therefore these parameters must be specified: the number of terms in the model, $m$; the sampling interval; the data ensemble length, $n$; and the prediction horizon, $b$. The aim of this paper is to recommend default numerical settings for these parameters that are suitable for most refinery control loops. 
It is unlikely that effort will be available to optimise the parameters individually for every loop in a refinery because the task would be similar in scope to the initial tuning of all the loops on installation of the original control system, i.e. a major undertaking. The benefit of default settings is that they bypasses this stage of the work. The proposed strategy for a large scale implementation relies upon a classification of control loops into generic types such as liquid flow, pressure or temperature-control loops. Data are collected from a few representative loops of each generic type and used to determine suitable default settings for the CLPA parameters. Alternatively the default settings reported in this paper can be applied directly because it is supposed that the CLPA parameters for representative examples of loops of one type can be applied to all loops of that type, an assumption that is justified later.

The use of defaults covers most cases and special cases can then be addressed individually. The recommended implementation strategy covers the following steps and is illustrated in Fig. 1.

- Apply the default settings and start routine CLPA.

- If many loops of a generic type cause exceptions then determine better values of the parameters.

- If a particular loop causes an exception then investigate whether better values of the parameters are needed for that loop or, if they are not, determine the cause of the loss of performance.

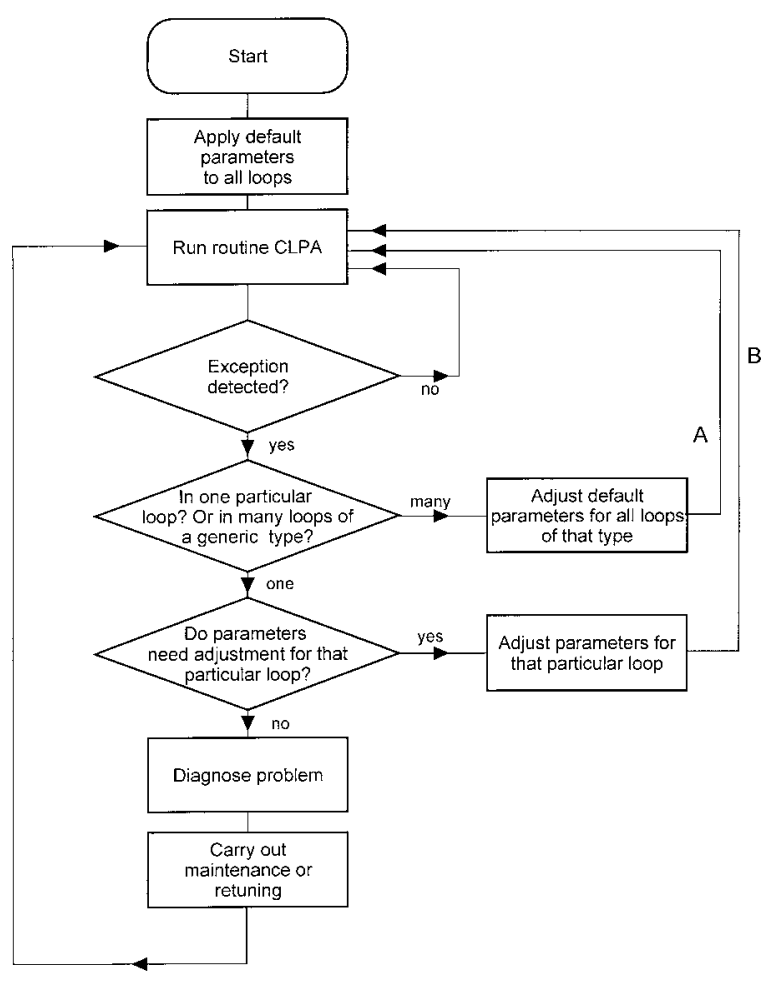

Fig. 1. The recommended strategy for refinery-wide implementation of control loop performance assessment.
The paths labelled A and B in Fig. 1 represent actions needed for loops for which the default parameters are not suitable. It is expected that as time passes the number of excursions around paths A and B would reduce rapidly.

\subsection{Refinery data sets}

The paper uses 12 refinery control loops as examples out of total of 41 that were examined during the implementation of CLPA. Table 1 lists the features of the 12 loops while Table 2 presents the controller error sequences and predictable components calculated using recommended parameters in the CLPA routines. The loops are located in refineries on three continents. The aim of showing a comparison across refineries is to illustrate that the same recommended default values apply to several refineries, not just to one.

All the CLPA assessments were made using data ensembles of 1400 samples or more, although for the sake of clarity only the first 200 samples are shown in Fig. 2. The engineering units are omitted from the vertical axes because it is the ratio between random and predictable components of the controller error that is of interest, not the absolute values.

Table 1 shows that Loops 1, 2, 4, and 8 had the highest CLPA values and are the worst performing of the 12 loops. Fig. 2 gives insight into what is wrong with them. Loops 1, 2 and 4 all have well-defined, oscillatory predictable components that are similar in magnitude to the controller error. By contrast Loops 5 and 12, for instance, have good (low) CLPA values. In those cases the predictable components are much smaller in amplitude than the controller error. That is, the controller error is mostly random in a loop with a good CLPA index. Loop 8 also has oscillations, but these are not detected by the predictable component. (Although the oscillations in controller error look by eye to be regular, a spectral analysis has shown that they are not). Rather, the problem with the performance of Loop 8 is that it has long excursions away from its set point, revealed by the fact that the controller error is persistently above or below zero for long periods of time. The predictable component reflects these long term deviations.

\subsection{Choice of sampling interval}

The choices of the sampling interval and the number of terms in the model are not independent of one another because they both influence the total time span captured by the autoregressive terms. The strategy used in this work was to choose the length of the autoregressive model to be the same for all cases $(m=30)$ and to adjust the sampling intervals for each generic type of loop. Reasons for the choice of a long time series and its numerical implications are discussed later in the paper. 
Table 1

Descriptions of twelve refinery control loops

\begin{tabular}{llcll}
\hline Loop & \multicolumn{1}{c}{ Type } & CLPA index & Comment & Location \\
\hline Loop 1: & Pressure & 0.498 & & Australia \\
Loop 2: & Pressure & 0.362 & Operator rated this loop as 'poor' & Australia \\
Loop 3: & Pressure & 0.096 & Operator rated this loop as 'good' & Australia \\
Loop 4: & Liquid flow & 0.358 & Loop used for tuning trials & UK \\
Loop 5: & Liquid flow & 0.159 & Loop 5 but with no pv filtering & UK \\
Loop 6: & Liquid flow & 0.021 & & UK \\
Loop 7: & Liquid flow & 0.136 & Known to be a well tuned loop & Australia \\
Loop 8: & Temperature & 0.408 & & Aus \\
Loop 9: & Temperature & 0.043 & & USA \\
Loop 10: & Temperature & 0.060 & & Australia \\
Loop 11: & Steam flow & 0.170 & & Australia \\
Loop 12: & Gas flow & 0.087 & &
\end{tabular}

Table 2

Recommended CLPA parameters for generic refinery loops

\begin{tabular}{lcc}
\hline Loop type & Sampling interval (s) & Prediction horizon, $b(\mathrm{~s})$ \\
\hline Pressure & 20 & 100 \\
Liquid flow & 10 & 30 \\
Temperature & $60-120$ & $360-600$ \\
Steam or gas flow & 60 & 300 \\
Level & 20 & 100 \\
\hline
\end{tabular}

Once the model length is fixed, the sampling interval can be chosen using plots like Fig. 3. An estimate of the closed loop impulse response is plotted, which shows visually the nature of the closed loop transient as the loop settles from an initial condition to its steady state (see Appendix). The objective is to select the sampling interval such that a typical closed loop impulse response is fully captured within 30 samples. In fact, the calculated CLPA index itself is not sensitive to the sampling interval provided the prediction horizon, $b$, is adjusted (i.e. if $b$ is 10 sample intervals for $1 \mathrm{~min}$ data it would be 5 sample intervals for 2 min data, see Loop 8 in Fig. 5, for instance). The reason for optimisation of the sampling interval relates to the diagnosis of the likely cause of a control loop problem. For instance, in a spectral analysis the spectral feature are properly resolved only when the sampling interval is correctly adjusted.

The estimated closed loop impulse response can itself be used as a diagnostic tool and its ability to resolve key features is influenced by the sampling interval. It is necessary to avoid both oversampling and undersampling. If the data are sampled too frequently the transient part of the closed loop impulse response does not settle within the 30 samples. If the data are undersampled, the closed loop impulse response is seen to settle within just a few samples and is not adequately captured because interesting features may be missed between samples.

Fig. 3 illustrates the choice of sampling interval for two loops. Fig. 3 shows the estimated impulse response of a pressure loop (Loop 1). In the upper figure, the sampling interval is $20 \mathrm{~s}$, while in the lower figure the sampling interval is $1 \mathrm{~min}$. In this case (and in the case of other pressure loops) the sampling interval of $20 \mathrm{~s}$ is chosen because the key features of the impulse response such as overshoot and the natural period of oscillation are well resolved. By contrast, the impulse response estimated from the $1 \mathrm{~min}$ sampling interval is all over within 10-12 sample intervals, leaving nothing but random movements for the next 18-20 sample intervals. This finding suggests a 1 min sampling interval would be too coarse for pressure loops.

Fig. 3 also shows the estimated impulse responses of a steam flow loop (Loop 11) at $20 \mathrm{~s}, 1 \mathrm{~min}$ and $3 \mathrm{~min}$ sampling intervals. Here, the $20 \mathrm{~s}$ samples do not capture all of the estimated impulse response. Using $1 \mathrm{~min}$ samples, however, the impulse response is fully captured; it can be seen to persist for about 23-25 samples. A sampling interval of $3 \mathrm{~min}$, by contrast, is too long, leaving the response poorly resolved. Therefore for Loop 11 and for other steam flow loops the recommended sampling interval is $1 \mathrm{~min}$. The choice of sampling interval can be confirmed by other means. A later sub-section shows the choice of the prediction horizon, $b$, has an interaction with the sampling interval. It will be seen that prediction horizon plots can confirm a good choice of sampling interval or reveal problems with a poor choice.

Table 2 shows the recommended sampling intervals for the generic types of refinery control loops.

\subsection{Choice of data ensemble length}

The data ensemble length influences the statistical confidence in the value of the CLPA index, which improves as the data ensemble length increases. Since the CLPA method uses the controller error and not the $p v$ it is not necessary for the loop to stay at the same set point throughout the period spanned by the data ensemble, but it is desirable that the loop characteristics 

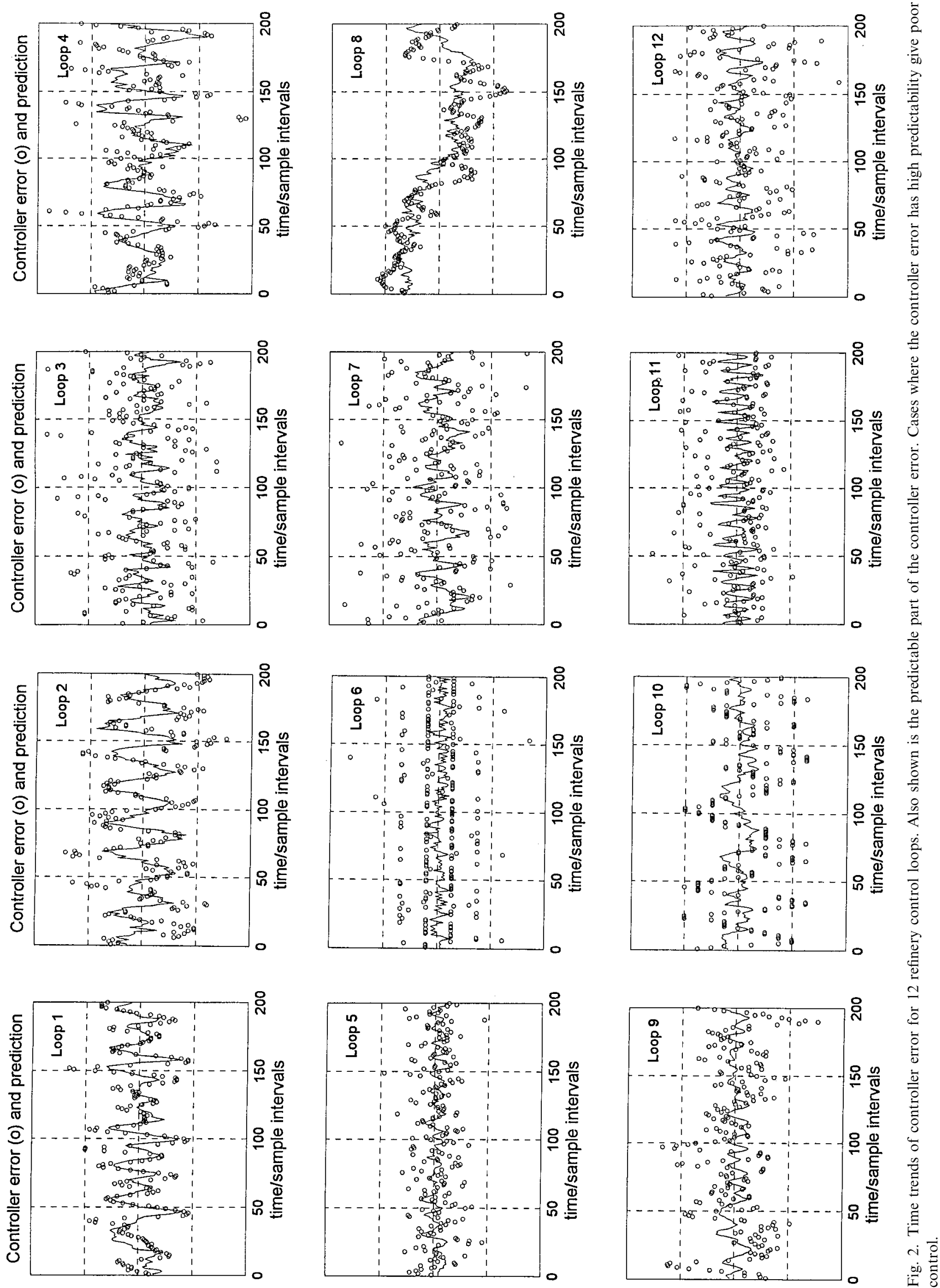

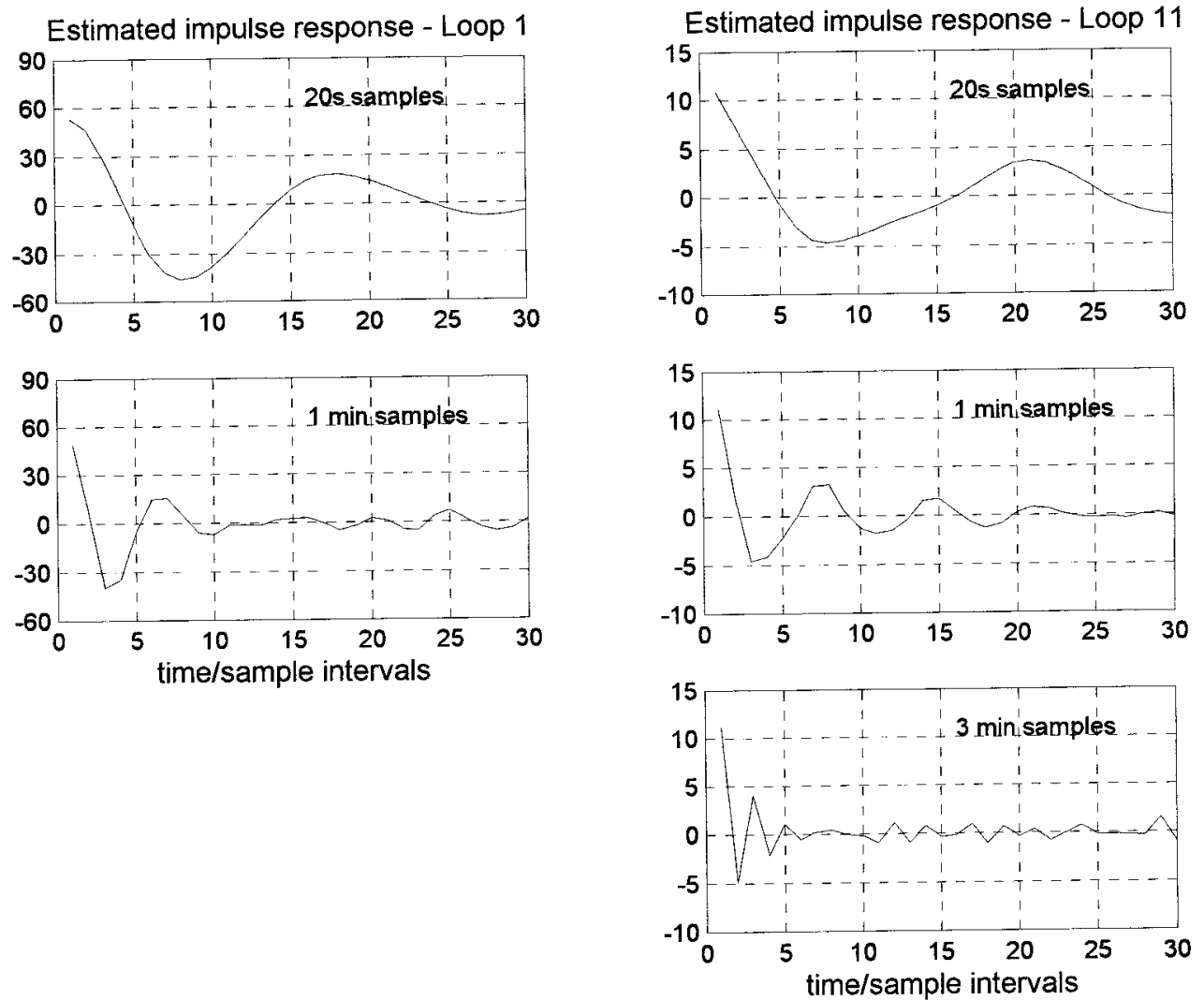

Fig. 3. Estimated impulse responses of Loops 1 and 11 with different sampling intervals. The sampling interval is selected that best captures the estimated impulse response within 30 samples.

stay the same. For example, the data ensemble should not contain an instrument recalibration episode or known plant disturbances such as feed switches or partial trips.

The effect of data ensemble length has been assessed using the confidence limits given in Desborough and Harris [7]. A good balance between statistical confidence and the steadiness of the loop characteristics is achieved with a data ensemble of 1500 samples. With the $10 \mathrm{~s}$ samples recommended for liquid flow loops the loop should thus be monitored for $4 \mathrm{~h}$ and $10 \mathrm{~min}$, while for $60 \mathrm{~s}$ samples as recommended for gas or steam flow the loop would need to be monitored for $25 \mathrm{~h}$. It is feasible to use shorter data ensembles of 1000 or even 500 samples, but the penalty is a broader confidence interval for the CLPA index.

Fig. 4 Shows the effect of short data ensembles using Loop 7 as an example. The upper figure shows the time trend of the controller error for 3000 samples. A feature of the trend is a brief episode of disturbance between samples 1200 and 1300 . When all 3000 points are used, the CLPA index is 0.136 with a standard deviation of 0.043. The lower four plots in Fig. 4 show the CLPA values and the standard deviations when shorter data ensembles are used. For instance, in the lower right hand plot the data ensembles are 300 points each. They have considerable variability and the error bars, which represent the standard deviations, are quite large. By contrast, the standard deviations for data ensemble of 1500 points are somewhat smaller.

In the plots for data ensembles of 1000, 500 and 300 samples it is clear that in each case the one which captures the episode of disturbance at sample 1200 has a significantly higher CLPA value. This effect demonstrates that shorter sequences are more responsive to changes in the loop's characteristics and that there is a trade-off between confidence in the CLPA value and its sensitivity to features in the data.

It is recommended that 1500 samples should normally be used, but shorter sequences of 1000 or even 500 samples still give a reasonable result and are more sensitive to brief episodes of disturbance. The use of data ensembles of 300 samples is not recommended because the scatter during normal running is relatively large and the standard deviations are high.

In Fig. 4 some of the plotted error bars reach below 0. The error bar truly reflects the standard deviation of the CLPA estimate, but is plotted presuming that the distribution is symmetrical in order to give a visual impression of the uncertainty in the CLPA values. The actual statistical distribution of CLPA values is not symmetrical, however, and is bounded below by zero. A second point of interpretation is that the standard deviation depends not only on the number of data 

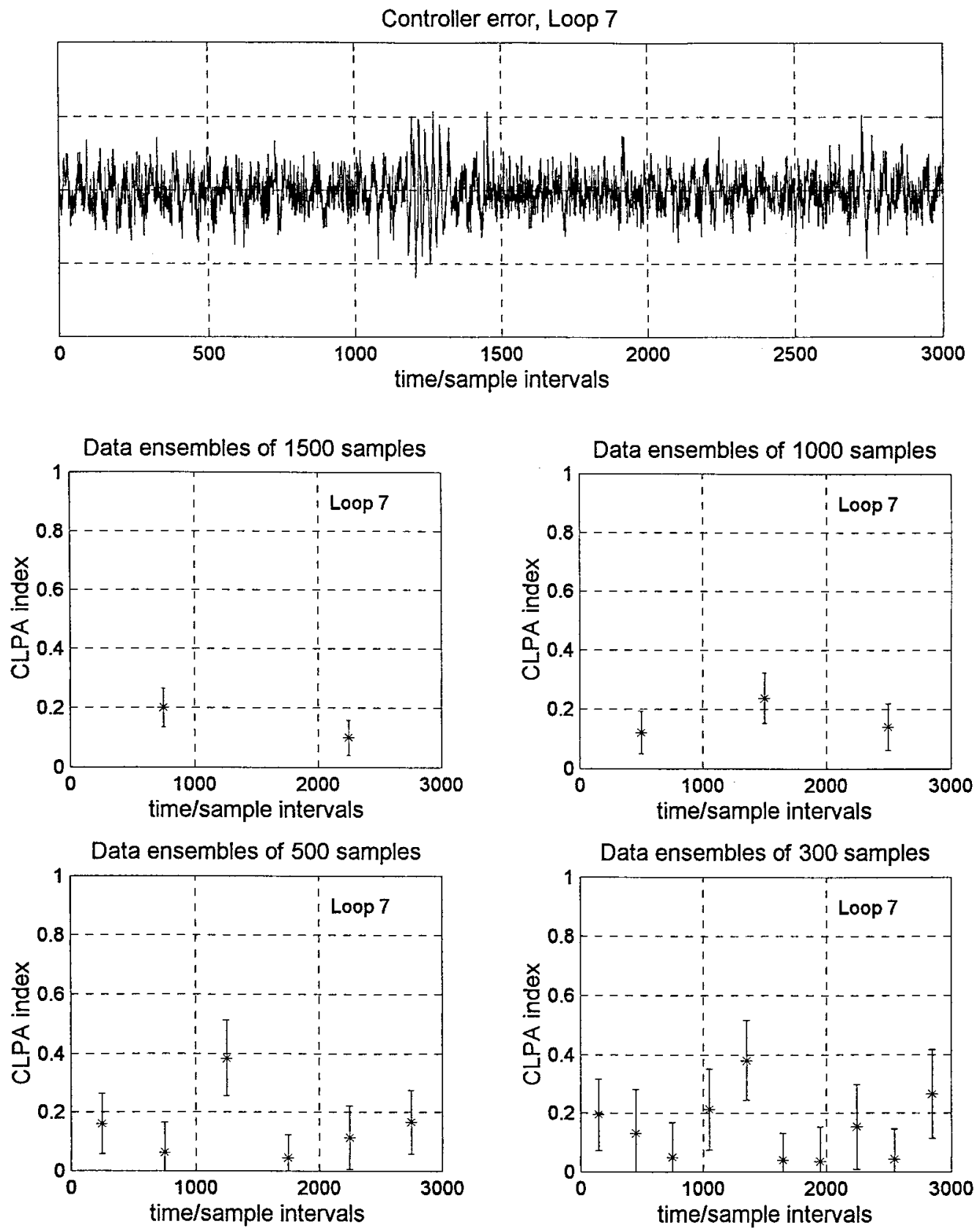

Fig. 4. The upper plot shows the time trend of the controller error for 3000 samples from Loop 7 . When all 3000 points are used, the CLPA index is 0.136 with a standard deviation of 0.043 . The lower four plots show the CLPA values and the standard deviations when shorter data ensembles are used.

points but also on the autocorrelation structure of the controller error sequence. For instance, the standard deviation for Loop 5 with 1500 samples (shown in Fig. 8) is smaller than that for Loop 7.

\subsection{Choice of prediction horizon}

Other authors have recommended that the prediction horizon be set to the process dead time. The reason is that a benchmark against which control performance can be assessed is minimum variance control, in which the elapsed time for restoration of control should be no more than the dead time of the process. MacGregor [18] discusses minimum variance process control in a manner that relates to the CLPA index while Harris, Desborough and Harris and Stanfelj et al. [6-8] developed the CLPA index as a minimum variance benchmark by equating the prediction horizon $b$ to the process dead time.

This prediction horizon need not, however, be the same as the process dead time. Desborough and Harris [7] discussed cases where the prediction horizon may be 
different from the dead time and Harris et al. [15] have commented on the theoretical consequences of a prediction horizon that is longer than the process dead time. Under these circumstances the benchmark is no longer minimum variance control and the quality of control in the interval between the dead time and the prediction horizon is not assessed.

It is time consuming and costly to determine dead times of control loops in an operating production process because the loops have to be taken loop off-line for step tests. Therefore a practical implementation of CLPA requires a means of determining a suitable prediction horizon from the closed loop data collected during normal process operations. Rather than reflecting the true dead time, it is recommended that the prediction horizon be treated as an engineering criterion, representing a demand made by the control engineer on the control loop. This demand is that the loop should deal with predictable components of the controller error within the specified prediction horizon.

Exploration of the effects of different choices of prediction horizon on a selection of representative loops gives an insight into a suitable horizon. Fig. 5 shows how the CLPA index varies with prediction horizon for the 12 refinery control loops. In each case an ensemble
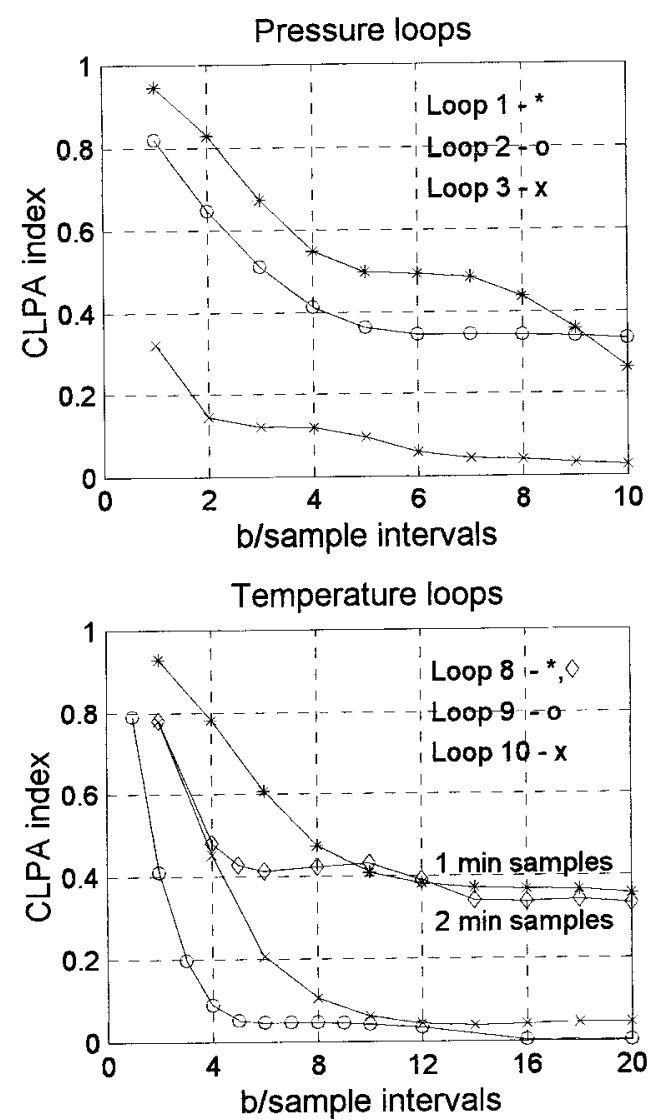

of data collected from the loop had its CLPA value assessed repeatedly for different choices of the parameter $b$ in Eq. (2). The form of these plots is interpreted in the 'Discussion' section. A good choice for the prediction horizon is one that falls on the plateau where the CLPA index does not vary rapidly. Recommendations for the prediction horizons of the generic types of refinery loops are given in Table 2.

\subsection{Choice of target CLPA value}

It is generally agreed that the performance target for process control should not be minimum variance control because the resulting aggressive actuator actions cause excessive wear of valves and may impose unacceptable disturbances on the process. The minimum variance controller can also be sensitive to model mismatch [19] such as that which might occur on a set point change in a mildly non-linear loop. If one insists that $b$ is the true process dead time then, when the requirement for minimum variance control is relaxed, the target for the CLPA index becomes some unspecified non-zero value. The target is also likely to be different for each loop. By contrast, the advantage of treating the prediction horizon as an engineering criterion
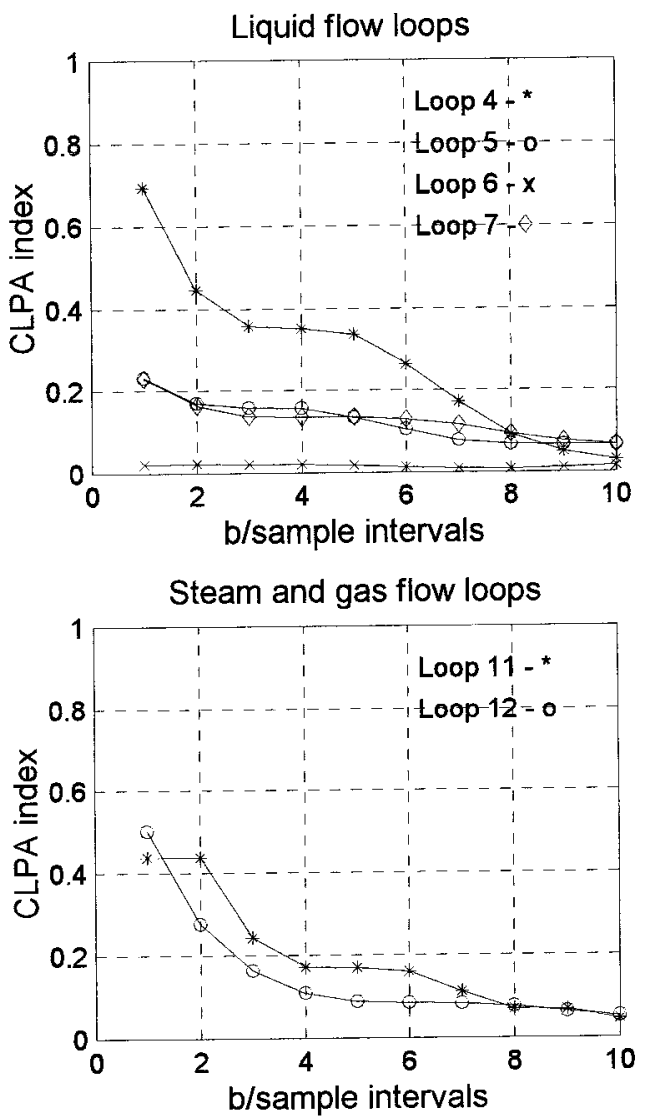

Fig. 5. Prediction horizon plots for 12 refinery control loops. In each case the CLPA index is calculated for a set of trial values of the prediction horizon, $b$ 
is that the ideal value of the CLPA index can be zero in all cases.

Experienced process operators can give guidance on which loops are well controlled or poorly controlled. As an example, the CLPA index has been calibrated against an operator's judgement for the pressure control loops 2 and 3. Loop 2, which had a CLPA index of 0.36, was judged unsatisfactory by the operator while Loop 3 having an index of 0.096 was considered good. Therefore, a CLPA index of 0.36 is taken to mean poor control. That the operator made such a judgement is unsurprising because Fig. 2 shows that Loop 2 has a well defined persistent oscillatory component in the controller error signal.

With a suitable choice of prediction horizon the target CLPA value should ideally be zero, as discussed above. Allowing for the effects of statistical variability and permitting some tolerance, a value of 0.15 or 0.1 is readily achievable and the target CLPA index should be set at, say, $\leqslant 0.15$.

It may also be useful to transform the index so that a well tuned loop has a maximum rather than a minimum value. The following transform gives a tuning index (TI) which is positive when the CLPA index is below 0.15 and equal to 100 when the CLPA index is 0 . Any negative value of this tuning index would be unacceptable.

$T I=\frac{0.15-\text { CLPA index }}{0.15} \times 100$

\subsection{Frequency of assessment and presentation of results}

Introduction of plant-wide CLPA into the refinery distributed control system (DCS) may pose a challenge due to limitations of acquisition bandwidth (i.e. the speed of data links), array handling and programmatic capability, and of real-time processing power. A choice has to be made to suit the site by the refinery control engineers in consultation with the DCS supplier. Implementation of the CLPA calculation in the plant information layer means data have to be transmitted through a gateway between the DCS and the plant information system. The advantages are that the CLPA routine can be implemented in a language of the user's choice, and that linkage to a spread sheet or a data base is easily implemented.

Putting the CLPA algorithm in the plant information layer, however, might preclude the continuous logging of every loop in the refinery at the fast rates required for CLPA because of the bandwidth requirements on the gateway. In that case the approach must be to schedule the CLPA assessments. The question then arises as to how frequently the assessments should be made. Some possible approaches are as follows; it is recommended that the decision should rest with the refinery control engineers because they need judgements about the priorities and economic values of different loops.

- All loops are considered to be of equal importance and are assessed as often as possible subject to the constraints of bandwidth.

- Some loops are given higher priority and are scheduled for assessment more often at the expense of other less important loops. Experience has shown that confining the CLPA system to economically important loops can reduce the number of monitored loops by more than $50 \%$.

- All loops for a given unit are assessed intensively while the rest of the refinery is ignored (e.g. prior to a turn-around, or during a performance audit).

Even in cases where there are no computing or bandwidth limitations it is not necessarily best to present the CLPA indexes continuously. The benefit of the CLPA index in refinery operations is that it focuses the control engineers' attention, so a system in which only exceptions are reported is appropriate. For instance, Harris et.al. [26] used a standard control chart to generate alarms and to detect changes in performance of a loop. The CLPA display screen should ideally highlight only those loops needing attention. For those loops, the control engineer should then be able to view further screens showing trends in the performance of the suspect loop over the past weeks and also additional signatures which give further insight into the nature of the problem. For instance, it is possible to distinguish oscillatory behaviour due to loop tuning from limit cycle oscillations due to non-linearities such as sticking valves [17]. The paper of Harris et. al. [26] has described such a user interface which also presents a summary of problem areas.

\section{Numerical implementation issues}

\subsection{Use of archived data}

Archived data are historical measurements from a process that have been retained in electronic form in a plant information system or data historian. The work reported here used the PI system (OSI Software Inc, San Leandro, CA). It is not advisable to use archived data for the purposes of CLPA analysis. The reason for caution is that archiving systems often modify the stored data. For example, the measurements may be smoothed to remove noise and compression may be applied both in order to keep up with increasing demands for stored information and to extract summary statistics [20].

Both smoothing and compression affect the calculated CLPA index. The purpose of smoothing is to 
reduce the effects of noise by averaging over previous measured values. The smoothed values thereby become correlated and the sequence is more predictable than the original sequence. Smoothing thus has an impact on the CLPA index because the index is a measure of predictability. Compression algorithms used in on-line data historians decide whether or not to archive a point using a variety of rules [21-23]. The rules are designed to capture the start and end of a trend and any exceptional values, but they are not designed to retain the noise and subtle predictable components that the CLPA index needs for its assessment.

Fig. 6 shows results for Loops 4 and 8 where a CLPA index has been calculated before and after smoothing. The smoothing filter has a first order form, and the amount of smoothing depends on a time constant, $\tau_{c}$, measured in sampling intervals. The quantities $p v_{s}$ are the smoothed values of the measured process variables, $p v_{m}$. Only the smoothed sequence $p v_{s}$ is stored in the archive:

$p v_{s}(n+1)=p v_{s}(n) \times \mathrm{e}^{-1 / \tau_{c}}+p v_{m}(n+1) \times\left(1-\mathrm{e}^{-1 / \tau c} c\right)$

Fig. 7 shows the impact of the 'Swinging door' [23] and the 'Box car and backward slope - BCBS' [22] methods of compression on Loops 4 and 8. The compression factor (horizontal axis) has the following interpretation: if one in five data points is retained after compression the compression factor is 5 , and so on. These illustrations show that both procedures alter the CLPA index in an unpredictable manner and that the use of compressed or smoothed data gives a misleading impression of the control loop performance. The conclusion is that if archived data are used for the CLPA assessment they should be identical with the original measurements, not smoothed or compressed.

Another type of digital filtering is also used in many control systems, as discussed in the next sub-section. Some control algorithms apply filtering to the process

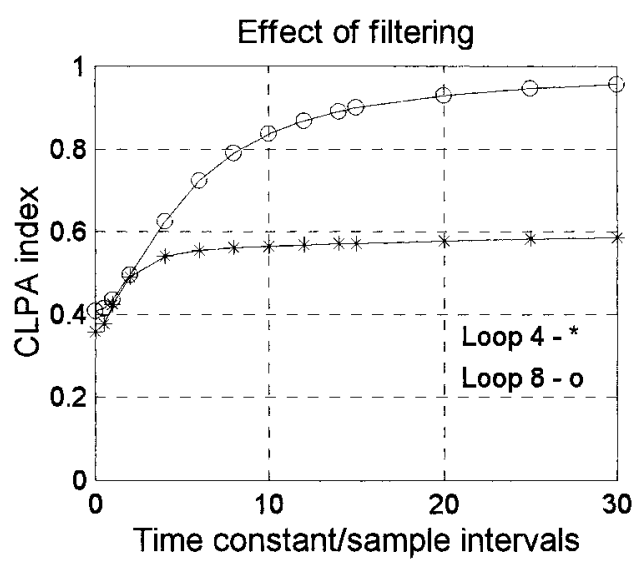

Fig. 6. Effects of smoothing the CLPA index. Smoothing filters of different time constants are applied to archived data. variable before using it in the feedback loop. Such realtime filtering is not a problem for a CLPA assessment because in that case the CLPA routine examines exactly the same data as used by the control loop itself.

\subsection{Use of quantized data}

Process variable measurements are generally quantized by a 10-bit analogue-to-digital converter. Many control algorithms, however, pre-filter the process variable before it is used by the feedback controller ( $p v$ filtering). Alternatively, the quantized signal may be used directly.

The presence of quantization affects the calculated CLPA index, however, because a quantized signal has rounding errors and is less predictable than the original continuous signal [24]. Therefore, a CLPA index determined for a quantized process variable can be misleading. Fig. 2 shows the controller error and predictable components for the same loop with and without $p v$ filtering (Loops 5 and 6). The least squares regression algorithm finds a smaller predictable component in the quantized data of Loop 6. The CLPA index for Loop 6 is thus smaller than in Loop 5, and Loop 6 appears to have superior performance even though the only difference is that $p v$ filtering has been switched off. Loop 10 is also quantized and also shows a smaller than expected predictable component.

A quantized regression algorithm designed specifically for recovering the continuous component from a quantized signal would give a better indication of the true performance of the loop. Such an algorithm is, however, more complicated to implement than linear autoregression because it uses a maximum-likelihood estimate rather than a least-squares method [25].

\subsection{Numerical conditioning}

The use of a long model with 30 autoregressive terms [Eq. (2)] can cause the matrix inversion in Eq. (3) to be close to singular, a situation that can arise if the data have good predictability. For instance, if the data have a periodic oscillation with no random component then the $k$ th row of $X$ would be identical to the $k+j$ th row, where $\mathrm{j}$ is the number of sample intervals in one cycle of the oscillation, so the matrix $\mathbf{X}^{T} \mathbf{X}$ would be singular. Data from normal process operations always have a noise component, however, which is usually enough to lift any degeneracy in the inversion of $\mathbf{X}^{T} \mathbf{X}$.

During refinery implementations the problem has been observed only twice. It has arisen in a case where a loop gave an oscillatory signal of large amplitude. The response became more and more predictable over the period of time that the loop performance deteriorated until the CLPA index exceeded 0.99. Then, on the next assessment, the algorithm gave a meaningless 

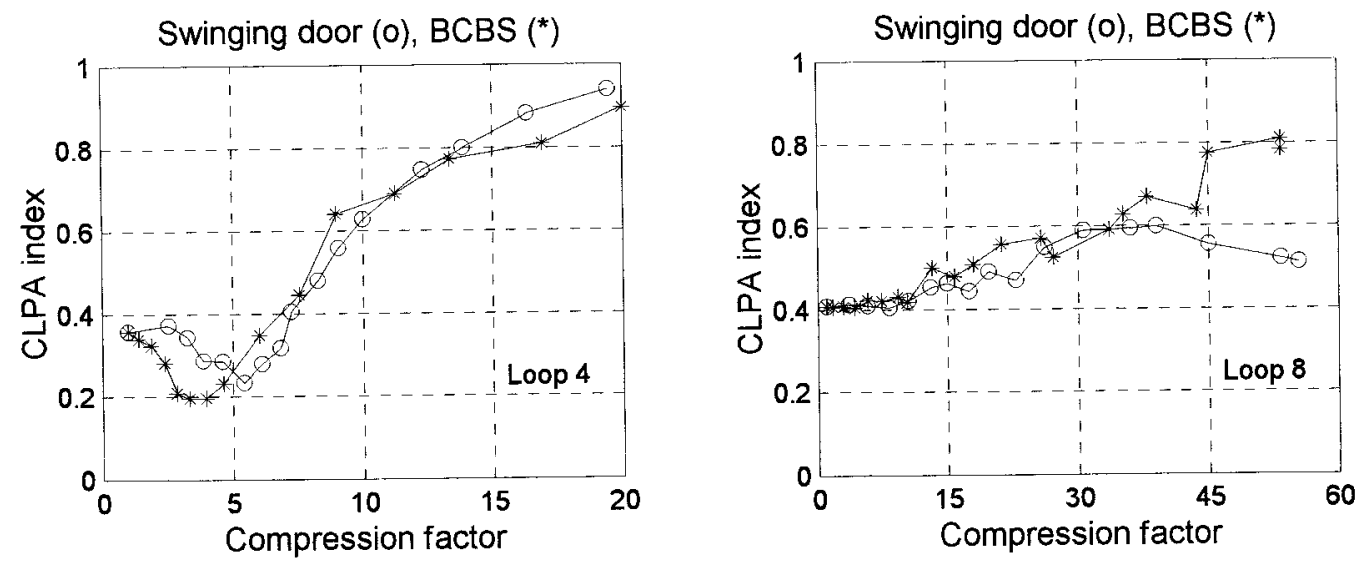

Fig. 7. The effects of the 'Swinging door' and 'BCBS' compression methods are compared. Different amounts of compression are applied to archived data.

negative CLPA index because the $\mathbf{X}^{T} \mathbf{X}$ inversion had become unstable. The problem has also arisen when using the CLPA algorithm after a method of compression that caused the data to become predictable over a long time scale.

The use of a long regression model leads to an additional numerical consideration, which is that the coefficients in such a model have high sensitivity. In particular, if the model were fitted to a second ensemble of data from the same loop it would be unlikely that the model coefficients would be the same. In practice this is not a problem because of the restricted use of the model. It is used only with the data ensemble for which it was identified and is never validated against unseen data. Its sole use is as the best fit to the predictable component within the set of data; the model coefficients are of no interest in themselves.

\subsection{Inclusion of an offset}

A key aim of modelling of the predictable component is to ensure that the residual sequence has a mean value of zero. That is to say, any steady-state offset should appear in the predictable component, $\hat{y}$, and not in the residuals.

One cause of an offset is the use of a proportionalonly controller with a non-integrating system. This situation can give a poor CLPA index because the offset between the set point and process variable has long term predictability. Use of the controller error sequence without mean centring ensures that a steady offset in the controller error appears in $\hat{y}$ and is classified as a loss of performance. If such a bias is not of interest the controller error sequence can be mean centred prior to the CLPA analysis.

A subtle effect of autoregression modelling can cause the residual sequence to have an unwanted non-zero mean value. A least squares fit of an autoregressive model without $a_{0}$ term will give a residual sequence with a non-zero mean if the average of the first $m$ samples is not exactly the same as the average of the remaining $n-m$ samples. The $a_{0}$ term, which is usually small, compensates for the difference. With the $a_{o}$ term included, the residual sequence has a mean value of zero and the predictable component then truly reflects any steady-state offset in the controller error sequence.

\section{Tuning trials}

Fig. 8 is a chart of the performance of loop 5 over an extended period of trials whose purpose was to illustrate that the controller tuning settings do indeed influence the CLPA index. The experiments were not aimed at optimising the tuning settings; to do that the refinery control engineers would use a commercial retuning tool. The episodes where the tuning settings changed are indicated on the figure. The trials returned several times to the base case settings to ensure that the basic performance of the loop had not changed. Each point on the figure used a data ensemble of $144010 \mathrm{~s}$ samples, representing $4 \mathrm{~h}$ running.

The PI tuning settings are given in Table 3 and relate to Algorithm A of Honeywell TDC3000 (Honeywell Industrial Automation and Control, Phoenix, AZ), which constructs the time trend $c(t)$ for the controller output from the time trend of controller error signal $y(t)$ (expressed as percent of range) according to the following expression:

$c(t)=K_{p}\left(y(t)+\frac{1}{\tau_{i}} \int y(t) \mathrm{d} t\right)$

Trials 1 and 2 represent the use of a P-only control. Since Loop 5 is for liquid flow it has no integration 


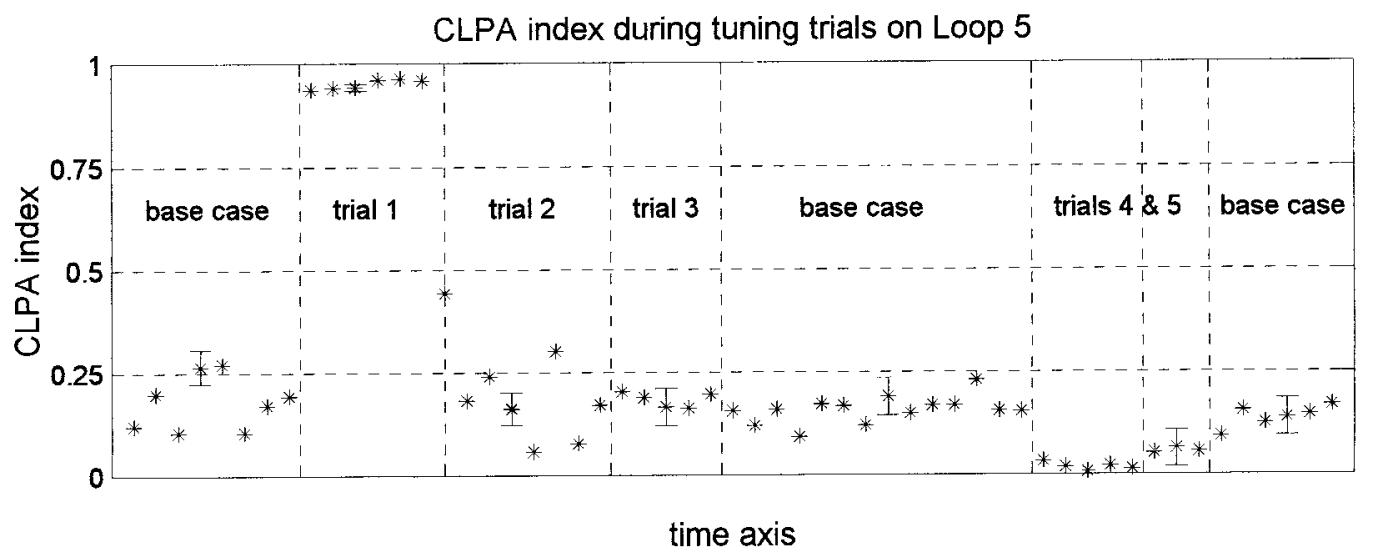

Fig. 8. CLPA results during tuning trials with Loop 5. Table 3 gives the settings for each trial.

Table 3

P-I controller settings for tuning trials on Loop 5

\begin{tabular}{lcccccc}
\hline Controller tuning & Base case & Trial 1 & Trial 2 & Trial 3 & Trial 4 & Trial 5 \\
\hline$K_{p}$ & 0.9 & 0.9 & 4.0 & 4.0 & 0.9 & 0.9 \\
$\tau_{i}$ (min) & 0.25 & P-only & P-only & 0.25 & 0.25 & 0.5 \\
$p v$ filtering & on & on & on & on & off & on \\
\hline
\end{tabular}

inherent in its dynamics, so P-only control gives an offset in the controller error whose size depends inversely in the controller gain. Therefore the performance is worse (the CLPA index is closer to 1) when the gain is low.

Trial 3 used a PI controller with a higher gain than the base case, which increased the natural resonant frequency of the loop. The reason why the natural frequency is of importance is that it is a common cause of a persistent oscillatory component. If a loop is resonant, it 'rings' at its natural frequency when excited by random instrument or process noise. However, although the frequency of oscillation was higher in trial 3 than in the base case, the amplitude of the oscillation was similar and the CLPA index was the same as in the base case. This example shows that loops with different characteristics can have the same CLPA index.

Trial 4 provided the data for Loop 6 . In this trial the tuning settings were the same as in the base case, but $p v$ filtering was off so that the output of the loop was quantized. As discussed earlier, quantized output gives the impression that the loop is performing better than it actually is. Trial 5 produced a genuine improvement on the base case by increasing the integration time. The effect of this in PI control of a loop whose open loop dynamics are dominated by a first order lag is to reduce the natural frequency and to increase the damping factor. That is, trial 5 has made the loop less resonant and has reduced the predictable oscillatory component. Fig. 9 shows the time trends and estimated impulse responses for Loop 5 with the base case and improved trial 5 settings.
For repair of a poorly performing loop it is usual practice in a refinery to take the loop off line for maintenance or for retuning using a commercial tuning package. Therefore fixing a poorly performing loop implies a loss of production and incurs costs in manhours. The CLPA performance index, together with further diagnosis, enables the control engineer make a good case to take a loop out of normal operation, and it focuses attention on loops likely to benefit from such attention.

\section{Discussion}

\subsection{Assumptions}

It is assumed that within a refinery most loops of a given generic type have similar closed loop responses provided they do not have hardware problems or structural problems such as control conflicts. For example, it is assumed that most refinery liquid flow loops can be assessed using the a sampling interval of $10 \mathrm{~s}$ and a prediction horizon of $30 \mathrm{~s}$, that most pressure loops can be assessed using $20 \mathrm{~s}$ samples and a prediction horizon of $100 \mathrm{~s}$, and so on.

Fig. 5 gives a justification for this assumption. The key features (the elbow and the plateau) show similarities even though the loops are located on three sites in different parts of the world.

Among the 41 refinery loops studied, a few had hardware problems such as sticking valves and for those the validity of the default settings could not be properly assessed. Of the rest, all were adequately handled by the recommended sampling intervals and prediction horizons except for one liquid flow loop that needed a shorter sampling interval.

The assumption of similar closed loop responses breaks down for equipment of a very different size such as small scale pilot plant. As reported below, the default settings for refinery liquid flow loops could not be 
Controller error (o) and prediction
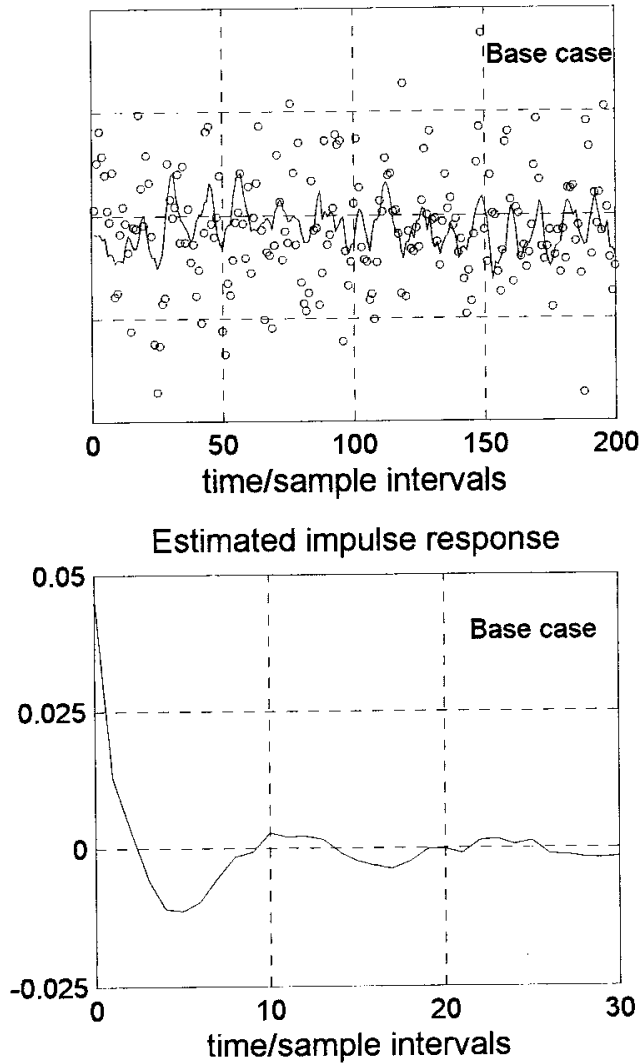

Controller error (o) and prediction
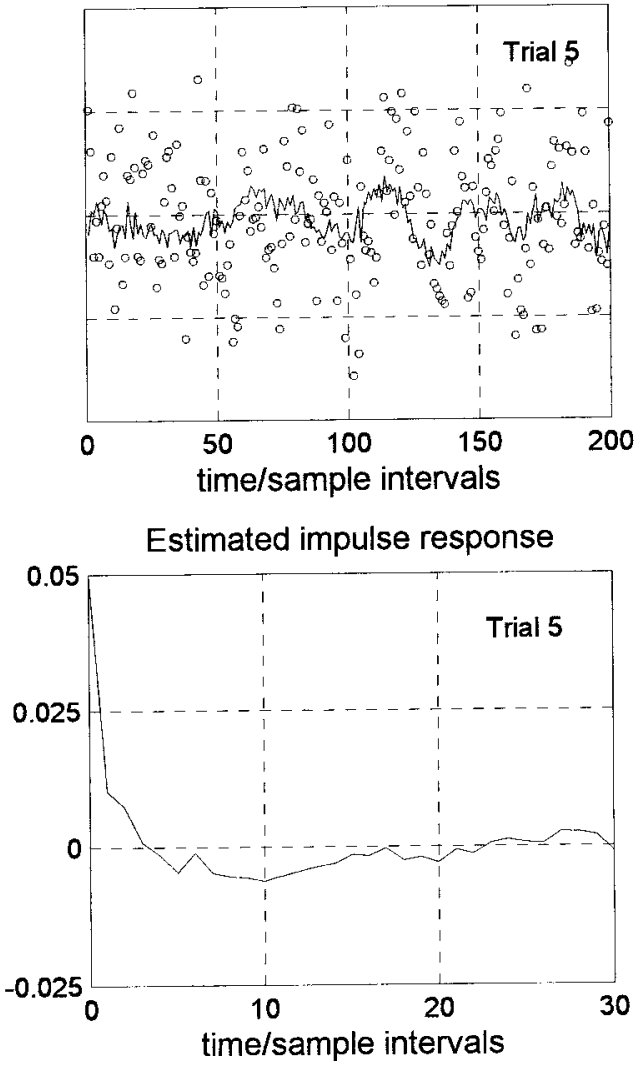

Fig. 9. Time trends and estimated impulse responses for Loop 5 with the base case and improved controller settings from trial 5.

applied in a university pilot plant. This finding emphasises that the default settings recommended in this paper are for refinery units, not for pilot plants.

\subsection{Interpretation of the prediction horizon plots}

The forms of the prediction horizon plots (Fig. 5) appear reproducible. Most of the plots show an initial rapid decline in the calculated CLPA index as the prediction horizon, $b$, increases. The majority of loops of all types then show an 'elbow' in this downward trend at which the downward trend stops and beyond which the CLPA index is steady. Moreover, for a given type of loop the elbow has been observed to occur at about the same value of the prediction horizon.

Understanding of the prediction horizon plots relates to the interpretation of the CLPA index as a 'predictability' index. When the CLPA index is close to 1 it means the predictable component is well defined, whereas when it is close to 0 the random residuals are dominant. The interpretation of the initial rapid reduction in the trend is that over a short time horizon the feedback acts to drive predictability out of the controller error signal.

Any plateau region where the index is constant means that the controller error contains a component that is predictable over a long time horizon. The value in this region depends on whether the loop is performing well or not. For instance, loop 1 has a high value on the plateau because this pressure loop is performing poorly.

If the controller error has a steady offset then the CLPA index can never attain a zero value because the average value of the controller error can be predicted over a long time scale. The same comment applies to a persistent oscillatory component in the controller error, which can also be predicted far ahead. A less persistent oscillation also shows a plateau, but then exhibits a further downward trend towards zero. That is to say, a less well defined oscillation loses coherence and cannot be predicted as far into the future.

It is the persistent predictable components that are of interest because they are the cause of the loss of performance. The recommendation, therefore, is to inspect plots like Fig. 5 for representative loops and to choose the prediction horizon so that it lies in the plateau region. In that way the CLPA examination will focus on the key problem, and moreover the calculated value of the index will be robust because the $d$ (index) $/ d b$ slope is small on the plateau.

The prediction horizon plots of Fig. 5 give an opportunity for a cross-check on the choice of sampling interval. In cases where the choice of sampling interval 
has been clear from a consideration of the estimated impulse response it has always been found that the best prediction horizons (on the plateau) are typically between three and 10 sampling intervals. Therefore, it is recommended that if the selected prediction horizon exceeds 10 sample intervals then an increase in the sampling interval should be considered. For instance, a 2 min sampling interval might be more suitable for Loop 8. With 1 min samples the plateau regions starts at 10 sample intervals $(600 \mathrm{~s})$. When the sampling interval is 2 min the plateau starts at five sample intervals (also $600 \mathrm{~s})$.

Loop 8 is not well tuned, however, and a decision to alter the default parameters should be treated with caution because retuning might be the more appropriate action. Loop 10 is a critical temperature loop that has had considerable attention from refinery control engineers. The position of its plateau shows the sampling interval of $1 \mathrm{~min}$ is well judged. Loop 10 suggests that if Loop 8 were better tuned not only would the value of the CLPA index on the plateau reduce but the elbow might move to the left.

\subsection{Why is the prediction horizon relatively longer in some cases?}

The table of generic default specifications shows that liquid flow loops have a prediction horizon of three sampling intervals while the prediction horizons for gas flow and pressure flow loops are five sampling intervals. A mundane reason why the ratios are not the same is that sampling intervals are convenient if they are multiples of $10 \mathrm{~s}$, or at least of some standard common factor. Thus if a liquid flow loop had the same ratio as pressure and gas flow with a prediction horizon of $30 \mathrm{~s}$ the ideal sampling interval would be $6 \mathrm{~s}$. Therefore $10 \mathrm{~s}$ is taken as the closest multiple of $10 \mathrm{~s}$.

The recommended prediction horizon for temperature loops is relatively long (5-10 sample intervals). One reason is that temperature loops usually operate in cascade mode, and the expectation for such loops is that they take longer to settle because the inner steam flow loop has dynamics of its own. The results in this article suggest that the sampling interval for the outer loop of a cascade has to be similar to that of the inner loop, but that the prediction horizon should be longer to reflect the slower dynamics of the outer loop.

Temperature loops may also vary in their time delays because of different layouts of piping, and this is a second reason why it has only been possible to suggest a range of suitable values for the sampling interval and the prediction horizon. The guideline suggested by Hägglund [3] could be applied; shorter prediction horizons could be used for temperature loops where smaller integration time has been used in the PI control algorithm in the past. The basis of this suggestion is that previous tuning activity would have used smaller (quicker) integral times for temperature loops with smaller time delays and quicker responses.

\subsection{Comment on the sampling interval for liquid flow}

It is a surprise that the selected sampling interval for liquid flow loops is as much as $10 \mathrm{~s}$, given that the valve stroke times are usually less than this. This observation raises two points. Firstly, it is the closed loop dynamics that are relevant in determining the CLPA sampling interval. The time constants of the closed loop are not the same as those of the open loop; fast open loop dynamics are often slowed down and slow open loop dynamics speeded up by the use of feedback. Another point is that the refinery units in the study were large units. It is to be expected that a study of the closed loop dynamics of smaller scale units or pilot plant would yield a different set of default parameters. Indeed, a small scale pilot plant study has shown an instance where $1 \mathrm{~s}$ sampling was needed for liquid flow.

If the $10 \mathrm{~s}$ sampling interval for refinery liquid flow loops causes concern it can be shortened to $6 \mathrm{~s}$, as mentioned previously. The recommended prediction horizon of $30 \mathrm{~s}$ would then be five sample intervals instead of three.

\section{Conclusions}

The paper has described practical experiences with the control loop performance assessment (CLPA) in a refinery-wide setting. Loops from refineries in Australia, USA and the UK have been included in the study.

The method, which is due to Desborough and Harris [7], is attractive because it uses data from normal process operations and does not require loops to be away from normal running for special tests. However, in a large process it is not feasible to tailor parameters of the algorithm to every individual control loop. Rather, the paper has recommended default parameter values after inspection of examples of each generic type of loop. The work has shown that the prediction horizon parameter in the CLPA algorithm can be set so that the analysis is sensitive to the persistent signals that cause loss of performance. The sampling interval for each type of loop and the data ensemble length have also been optimised.

Issues concerning numerical implementation have been examined. In particular, the work has shown that the use of CLPA with archived data should be avoided because smoothing and compression of archived data affect the results. Cases where the process variable $(p v)$ measurements are quantized by analogue-to-digital conversion have been shown to give a misleadingly good value of the performance index. That is, the performance of a loop with a quantized $p v$ is over-estimated. 
The value of a refinery-wide implementation of CLPA is that it can routinely monitor individual control loops and focus on exceptions that need attention. It helps a control engineer to build a case for maintenance of the control loop and to estimate the benefits to arise from maintenance. Only if the basic single-input-singleoutput loops are performing well can the full potential of advanced multivariable control and optimisation be realised.

\section{Acknowledgements}

The authors thank John Davis, Mike Knight, Peter Prichard, Doug Rothenberg and Bruce Varley of BP Oil for directing the work and managing the refinery visits. Thanks also to Roger Lambert and John Warren of BP Oil, Grangemouth Refinery Ltd, as well as to Robert Sadowski and Bradley Scarpness for technical input and support. Nina Thornhill gratefully acknowledges the financial support of a Royal Society Industry Fellowship.

\section{Appendix A. Estimated closed loop response}

The following comments give an insight into the cross-correlation of the modelling residuals from (1) and the controller errors, $y$, as an estimate of the closed loop impulse response [27]. The autoregressive model

$$
\begin{aligned}
y(i+b) & =a_{o}+a_{1} y(i) \\
& +a_{2} y(i-1)+\ldots+a_{m} y(i-m+1)+r(i+b)
\end{aligned}
$$

is taken to be equivalent to a moving average model:

$$
\begin{aligned}
y(i+b)=c_{0} n(i+b) & +c_{1} n(i+b-1) \\
& +c_{2} n(i+b-2)+\ldots
\end{aligned}
$$

where the terms $n(i+b)$ and so on are a white noise sequence. More compactly, the autoregressive model can be expressed in the notation of the delay operator $q^{-1}$ as:

$A\left(q^{-1}\right) y(i+b)=r(i+b)$

while the moving average model is:

$$
y(i+b)=C\left(q^{-1}\right) n(i+b)
$$

If the $A\left(q^{-1}\right)$ polynomial is long enough then $C\left(q^{-1}\right) \approx\left(A\left(q^{-1}\right)\right)^{-1}$ and the white noise sequence can be taken as identical to the residuals $r(i+b)$. In that case the coefficients $c_{0}, c_{1}$ and so on comprise the impulse response of the closed loop system. It is not necessary to perform the inversion of $A\left(q^{-1}\right)$. Rather, the closed loop impulse response is identified from the cross-correlation function of the residuals $r$ with the controller errors $y$ :

$c_{k}=\frac{1}{n-k} \sum_{i=k}^{n} r(i-k) y(i)$

The impulse response sequence calculated in the above manner should be used with caution. It presumes that the dynamics captured in $C\left(q^{-1}\right)$ are the same as those of the closed loop transfer function from $s p$ to $p v$. There are cases, especially in loops where the $p v$ is dominated by quantization error, when this assumption is not true.

\section{References}

[1] K.J. Åström, Assessment of achievable performance of simple feedback loops, Int. J. Adap. Control and Sig. Proc. 5, (1991) 319.

[2] D.B. Ender, Process control performance: not as good as you think, Control Engineering Sept, (1993), 180-190.

[3] T. Hägglund, A control-loop performance monitor, Control Eng. Practice 3, (1995) 1543-1551.

[4] G.D. Martin, L.E. Turpin, R.P. Cline, Estimating control function benefits. Hydrocarbon Processing, June, (1991), 68-73.

[5] D. Kozub. Controller performance monitoring and diagnosis, Proceedings of Fifth International Conference on Chemical Process Control, CACHE Corp, 1997 pp. 83-96.

[6] T.J. Harris, Assessment of control loop performance, Can. J. Chem. Eng. 67, (1989) 856-861.

[7] L. Desborough, T. Harris, Performance assessment measures for univariate feedback control, Can. J. Chem. Eng. 70, (1992) 11861197.

[8] N. Stanfelj, T.E. Marlin, J.F. MacGregor, Monitoring and diagnosing process control performance: the single loop case, Ind. Eng. Chem. Res. 32, (1993) 301-314.

[9] D.J. Kozub, C.E. Garcia, Monitoring and diagnosis of automated controllers in chemical process industries. AIChE Meeting, St Louis, MI, 1993.

[10] N.F. Thornhill, R. Sadowski, J.R. Davis, P. Fedenczuk, M.J. Knight, P. Prichard, D. Rothenberg, Practical experiences in refinery control loop performance assessment, IEE Conference Publication 427/1, (1996) 175-180.

[11] M. Perrier, A.A. Roche, Towards mill-wide evaluation of control loop performance. Proc. Control. Syst. '92, Whistler, BC, 1992.

[12] C.B. Lynch, G.A. Dumont, Control loop performance monitoring, IEEE Trans. Control Systems Technol 4, (1996) 185-92.

[13] P.J. Jofriet W.L. Bialkowski, Process knowledge: the key to online monitoring of process variability and control loop performance. Proc. Control. Syst. 96, Halifax, NS, (1996) 187-193.

[14] J.G. Owen, D. Read, H. Blekkenhorst, A.A. Roche, A mill prototype for automatic monitoring of control loop performance, Proc. Control. Syst. 96, Halifax, NS, 1996, pp. 171-178.

[15] T.J. Harris, F. Boudreau, J.F. MacGregor, Performance assessment of multivariable feedback controllers, Automatica 32, (1996) 1505-1518.

[16] P.J. Jofriet, C.T. Seppal, B.W. Surgenor, T.J. Harris, An expert system for control loop performance, Pulp \& Paper Canada 97, (1996) 207-211. 
[17] N.F. Thornhill, T. Hägglund, Detection and diagnosis of oscillation in control loops, Control Eng. Prac. 5, (1997) 1343-1354.

[18] J.F. MacGregor, On-line statistical process control. Chem. Eng. Prog. October (1988) 21-31.

[19] K.J. Åström, Introduction to Stochastic Control Theory, Academic Press, New York, 1970.

[20] J.P. Kennedy, Data treatment and applications - future of the desktop, Proceedings of Foundations of Computer aided Process Operations, CACHE Corp, 1993.

[21] R.S.H. Mah, A.C. Tamhane, S.H. Tung, A.N. Patel, Process trending with piecewise linear smoothing, Comput. Chem. Engng. 19, (1995) 129-137.

[22] J.C. Hale, H.L. Sellars, Historical data recording for process computers, Chem. Eng. Prog. November (1981) 38-43.
[23] E.H. Bristol, Swinging door trending: adaptive trend recording? ISA National Conf. Proc. (1990) 749-753.

[24] R.E. Curry, Estimation and Control with Quantized Measurements, Research Monograph 60, MIT Press, Cambridge, MA, 1970.

[25] I. Ziskand, D. Hertz, Multiple frequencies and AR parameters estimation from one bit quantized signal via the EM algorithm, IEEE Trans. Sig. Proc. 11, (1993) 3202-3206..

[26] T.J. Harris, C.T. Seppala, P.J. Jofreit, B.W. Surgenor, Plant-wide feedback control performance assessment using an expert system framework, Control Engng. Practice 4, (1996) 1297-1303.

[27] M.L. Tyler, M. Morari, Performance monitoring of controlsystems using likelihood methods, Automatica 32, (1996) 11451162. 